\title{
GEOMETRIC WAVELET TRANSFORM FOR OPTICAL FLOW ESTIMATION ALGORITHM
}

\author{
Cheriet Leila ${ }^{1}$, Chenikher Salah ${ }^{2}$ and Boukari karima ${ }^{3}$ \\ ${ }^{1}$ Department of Electronic, Annaba University, Annaba, Algeria \\ ${ }^{2}$ Department of Genie Electric, Tebessa University, Tebessa, Algeria \\ ${ }^{3}$ Department of Electronic, Annaba University, Annaba, Algeria
}

\begin{abstract}
This paper described an algorithm for computing the optical flow (OF) vector of a moving objet in a video sequence based on geometric wavelet transform (GWT). This method tries to calculate the motion between two successive frames by using a GWT. It consists to project the OF vectors on a basis of geometric wavelet. Using GWT for OF estimation has been attracting much attention. This approach takes advantage of the geometric wavelet filter property and requires only two frames. This algorithm is fast and able to estimate the OF with a low-complexity. The technique is suitable for video compression, and can be used for stereo vision and image registration.
\end{abstract}

\section{KEYWORDS}

Geometric Wavelet, Curvelet Wavelet, Motion Estimation, Optical Flow

\section{INTRODUCTION}

Recherch work on optical flow estimation has previously been approved out to different applications which found in various fields such as signal and image processing; pattern recognition and computer vision, astronomy, acoustics and geophysics. It also finds place in medicine and meteorology by processing related images. Another area of high importance is the detection and tracking of moving targets in military applications [8],[9],[10] and [12].

A Great number of approaches for OF estimation have been proposed in the literature, including gradient-based, correlation-based, energy based, and phase based techniques [1].

The use of real wavelet in this approach suffers from two main problems; the lacks of shift invariance and poor directional selectivity. The geometric wavelet solves these problems and still desired to provide special characteristics.

The wavelet curvature is applicable principally in motion estimation edge detection and texture discrimination. 
The rest of the paper is organized as follows. In Section 2, we introduce the principle and the description of the proposed algorithm. Section 3 shows the experimental performance of optical flow estimation. Finally, Section 4 concludes our contribution and merits of this work.

\section{OPTICAL FLOW ESTIMATION ALGORITHM}

\subsection{Geometric Wavelet}

Curvelet transform correspond on association of different steps: the application of filters passbands, on segmentation dyadic of each band frequency and transform Ridgelet on each zone segmented. The decomposition frequented associated on the dyadic segmentation permit conditioner the data for the Ridgelet transform with the aim of describe the singularities, where the size and the form of the motif variant. The description of the singularities where the size and the form of the motif variant. The description correspond the variability on the position: position associates on different dyadic zones.

The aim of this variant is permit the separation in the signal where the motif is very complex that in Ridgelet transform. This transform is very adapted for the split of a real image, it approximate the contour from an ensemble of segments. This transform ameliorant the singularities of an image, on the contrary, it is very redundant. It exists the alternatives for the limitation of the redundancy.

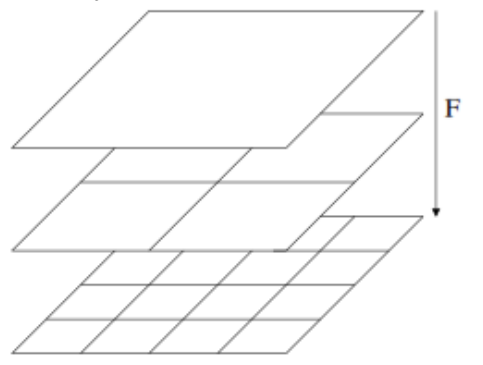

(a)

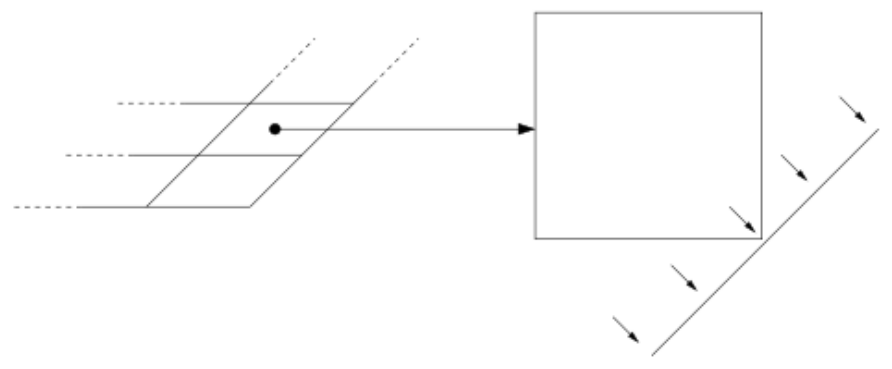

(b)

Figure (1): (a) Decomposition of an image with filter banc. (b) Application of Ridgelet transform in each zone dyadic.

\subsection{Gradient Constraint}

Our work shows that geometric wavelet transform technique seems more accurate in optical flow estimation.

This technique is based on the assumption of the gradient constraint. This equation can be expressed as

$I(x+\Delta x, y+\Delta y, t+\Delta t)=I(x, y, t)+\frac{\partial I}{\partial x} \Delta x+\frac{\partial I}{\partial y} \Delta y+\frac{\partial I}{\partial t} \Delta t+H . O . T$

$\frac{\partial I}{\partial x} \Delta x+\frac{\partial I}{\partial y} \Delta y+\frac{\partial I}{\partial t} \Delta t=0$ 
Or

$\frac{\partial I}{\partial x} \frac{\Delta x}{\Delta x}+\frac{\partial I}{\partial y} \frac{\Delta y}{\Delta t}+\frac{\partial I}{\partial t} \frac{\Delta t}{\Delta t}=0$

Which result in:

Thus:

$$
\frac{\partial I}{\partial x} V_{x}+\frac{\partial I}{\partial y} V_{y}+\frac{\partial I}{\partial t}=0
$$

$I_{x} V_{x}+I_{y} V_{y}=-I_{t}$

$\nabla I^{T} \cdot \vec{V}=-I_{t}$

Where $V_{x}, V_{y}$ are the optical flow of $I(x, y, t)$ and $\frac{\partial I}{\partial x}, \frac{\partial I}{\partial y}$ and $\frac{\partial I}{\partial t}$ or $I_{x}, I_{y}$ and $I_{t}$ are the derivatives spatial and temporal of the image.

Our method introduces an additional condition for estimating the optical flow because it is an equation in two unknowns and cannot be solved as such. To discover the optical flow another set of equations is needed, given by some additional constraint.

\subsection{Method}

The framework of the algorithm is illustrated:

$$
\begin{gathered}
I_{X} \cdot f(X, Y) p=-I_{t} \\
f(X, Y)=\left[\begin{array}{rrrrrrr} 
& \ldots & & & \ldots & \\
x_{i} & y_{i} & 1 & 0 & 0 & 0 \\
0 & 0 & 0 & x_{i} & y_{i} & 1 \\
& \ldots & & & \cdots &
\end{array}\right]^{T},
\end{gathered}
$$

Where

$$
I_{X}=\left[\begin{array}{ccccccc}
I_{x_{1}} & I_{y_{1}} & 0 & 0 & \cdots & 0 & 0 \\
\vdots & \vdots & \vdots & \vdots & \ddots & 0 & 0 \\
0 & 0 & 0 & 0 & \cdots & I_{x_{M}} & I_{y_{M}}
\end{array}\right]
$$

By applying the geometric wavelet transforms at the level $l$, we get the hierarchical gradient constraint functions (see figure 1).

$A^{l} p=-I_{t}^{l}$

The global equation of gradient constraint at all scale levels $L$ is:

$A p=b$

$A=\left[A^{0}, A^{1}, \ldots, A^{L}\right] \quad b=-\left(I_{t}^{0}, I_{t}^{1}, \ldots I_{t}^{L}\right)^{T}$ 


\section{Experiment results:}

In our experiments, we used three of sequences synthetic and four methods for comparison. For our simulation the low and high pass filter are done by

$$
\begin{gathered}
h_{\text {low }}=[-0.5-j, 3-0.5 j,-0.5+j, 3+0.5 j] \\
h_{\text {high }}=[-1.5-j, 1-0.5 j,-1.5+j, 1+0.5 j]
\end{gathered}
$$

We evaluate the optical flow by using the angular error measurement between the correct velocity $\left(u_{c}, v_{c}\right)$ and the estimate velocity $\left(u_{e}, v_{e}\right)$ with $100 \%$ density, the average error and standard deviation were calculated.

Three images sequences were used to test our algorithm and compared with other optical flow technique:

$$
\theta_{e r r}=\arccos \left(\frac{u_{e} u_{c+} v_{e} v_{c}+1}{\sqrt{u_{e}^{2}+v_{e}^{2}+1} \sqrt{u_{c}^{2}+v_{c}^{2}+1}}\right)
$$

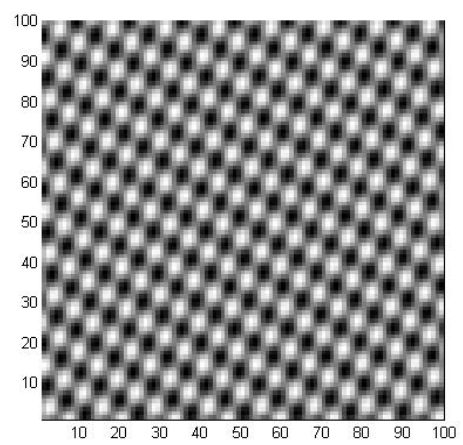

Figure 2.A. Sequence mysineB-6

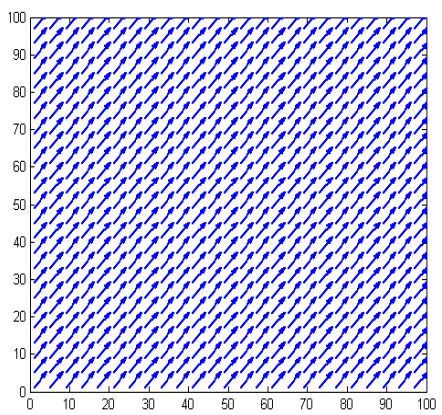

Figure 2.B. Optical Flow measuring by using real wavelet transforms 
International Journal of Computer Graphics \& Animation (IJCGA) Vol.4, No.2, April 2014

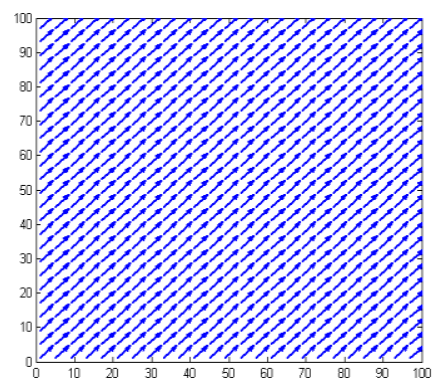

Figure 2C. Optical Flow measuring by using geometric wavelet transforms

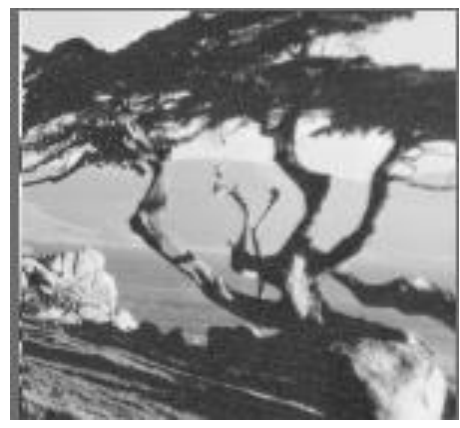

Figure 3.A. Sequence Diverging tree

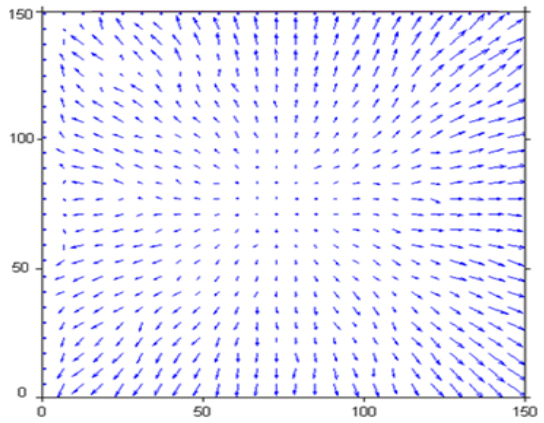

Figure 3.B. Optical Flow measuring by using real wavelet transforms

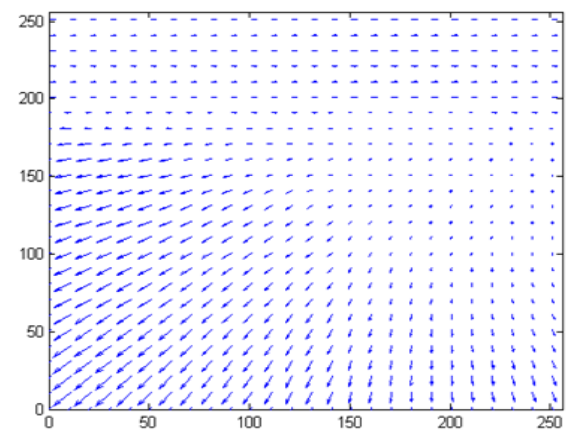

Figure 3C. Optical Flow measuring by using Geometric wavelet transforms 
International Journal of Computer Graphics \& Animation (IJCGA) Vol.4, No.2, April 2014

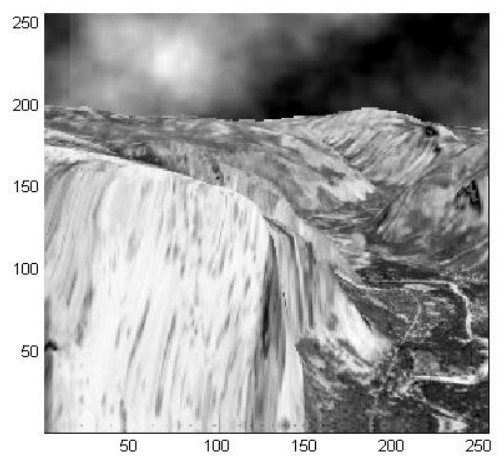

Figure 4.A. Sequence Yosemite

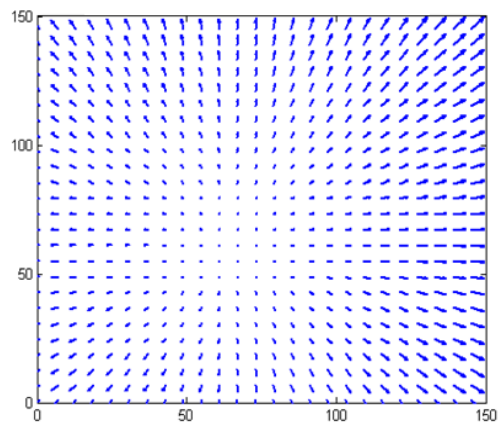

Figure 4.B. Optical Flow measuring by using real wavelet transforms

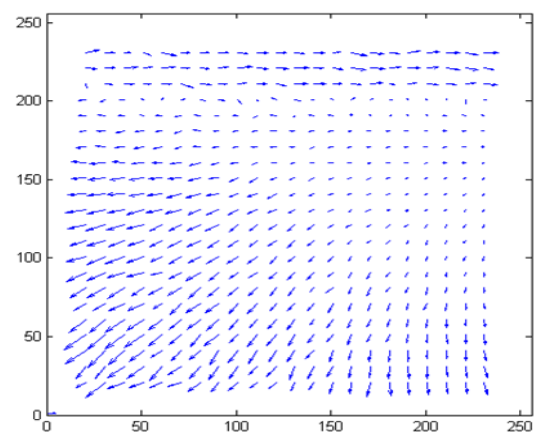

Figure 4C. Optical Flow measuring by using Geometric wavelet transforms

Table 1.Comparison of different methods of sequence "my sineB.6".

\begin{tabular}{|c|c|c|c|c|}
\hline Method & Images & Error & deviation & Density (\%) \\
\hline $\begin{array}{c}\text { Motion estimation using complex } \\
\text { wavelet }\end{array}$ & 2 & $0.79^{\circ}$ & 0.68 & 100 \\
\hline Motion estimation using real wavelet & 2 & $5.35^{\circ}$ & $4.56^{\circ}$ & 100 \\
\hline Horn et Schunk (original) & 2 & $12.02^{\circ}$ & $11.72^{\circ}$ & 100 \\
\hline Horn et Schunk (modify) & $7-13$ & $2.55^{\circ}$ & $3.67^{\circ}$ & 100 \\
\hline Anandan & 2 & $7.64^{\circ}$ & $4.96^{\circ}$ & 100 \\
\hline Singh & 2 & $8.60^{\circ}$ & $4.78^{\circ}$ & 100 \\
\hline
\end{tabular}


International Journal of Computer Graphics \& Animation (IJCGA) Vol.4, No.2, April 2014

Table 2.Comparison of different methods of sequence "Diverging tree".

\begin{tabular}{|c|c|c|c|c|}
\hline Method & Images & Error & deviation & Density (\%) \\
\hline Motion estimation using complex wavelet & 2 & $0.79^{\circ}$ & 0.68 & 100 \\
\hline Motion estimation using real wavelet & 2 & $5.35^{\circ}$ & $4.56^{\circ}$ & 100 \\
\hline Horn et Schunk (original) & 2 & $12.02^{\circ}$ & $11.72^{\circ}$ & 100 \\
\hline Horn et Schunk (modify) & $7-13$ & $2.55^{\circ}$ & $3.67^{\circ}$ & 100 \\
\hline Anandan & 2 & $7.64^{\circ}$ & $4.96^{\circ}$ & 100 \\
\hline Singh & 2 & $8.60^{\circ}$ & $4.78^{\circ}$ & 100 \\
\hline
\end{tabular}

Table 3.Comparison of different methods of sequence "Yosemite".

\begin{tabular}{|c|c|c|c|c|}
\hline Method & Images & Error & deviation & Density (\%) \\
\hline $\begin{array}{c}\text { Motion estimation using complex } \\
\text { wavelet }\end{array}$ & 2 & $4.52^{\circ}$ & $4.65^{\circ}$ & 100 \\
\hline $\begin{array}{c}\text { Motion estimation using real wavelet } \\
\text { Horn et Schunk (original) }\end{array}$ & 2 & $9.43^{\circ}$ & $8.87^{\circ}$ & 100 \\
\hline Horn et Schunk (modify) & $7-13$ & $11.26^{\circ}$ & $10.59^{\circ}$ & 100 \\
\hline Anandan & 2 & $15.84^{\circ}$ & $13.46^{\circ}$ & 100 \\
\hline Singh & 2 & $13.16^{\circ}$ & $12.07^{\circ}$ & 100 \\
\hline
\end{tabular}

\section{Conclusion}

The algorithm estimate the OF by resolving the resulted equations from the projection of the gradient constraint in the Geometric wavelet bases. The experimental results demonstrate that the

Geometric wavelet is capable to estimate many kinds of movement. Testing on synthetic data sets has shown good performance compared par real wavelet.

\section{REFERENCES}

[1] Lee, S.hyun. \& Kim Mi Na, (2008) "This is my paper", ABC Transactions on ECE, Vol. 10, No. 5, pp120-122.

[2] Gizem, Aksahya \& Ayese, Ozcan (2009) Coomunications \& Networks, Network Books, ABC Publishers.

genetic trail bounded approximation for H. 264/AVC codecs- MA Haque - Multimedia Tools and Applications, 2012 - Springer

[1] Models for Local Optical Flow Estimation- T Corpetti - Scale Space and Variational Methods in Computer, 2012 - Springer.

[2] S.LEE, Fast motion estimation based on search range adjustment and matching point decimation, Department of Imaging Engineering, ChungAng University Seoul, COREE, REPUBLIQUE DE, 2010.

[3] Large Displacement Optical Flow: Descriptor Matching in Variational Motion Estimation Brox,

[4] T.; Malik, J.; Dept. of Electr. Eng Comput. Sci.,Univ. of California at Berkeley, Berkeley, CA, USA2011 
International Journal of Computer Graphics \& Animation (IJCGA) Vol.4, No.2, April 2014

[5] A.Ahmadi, M.M.Azadfar, "Implementation of fast motion estimation algorithms comparison with full search method in H.264", IJCSNS International Journal of Computer Science and Network Security, VOL.8 No.3, March 2008

[6] J.Barron, D.Fleet, and S. Beauchemin. "Performance of optical flow techniques". Int. J. Computer Vision, 1994.

[7] W Lawton, 'Applications of Complex Valued Wavelet Transforms to Subband Decomposition', IEEE Trans. Sig. Proc., 41, 12, 3566-3568, 1993.

[8] N G Kingsbury, 'Image Processing with Complex Wavelets', Phil. Trans. Royal Society London, 1999 